\title{
EL USO DEL SUBJUNTIVO/INDICATIVO CON EL OPERADOR DE MODALIDAD QUIZÁ(S): ANÁLISIS DEL CORPUS
}

\author{
Miroslava AUROVÁ \\ Universidad de Bohemia del Sur, České Budějovice
}

\begin{abstract}
En): The present article deals with the modality operator quizás in Spanish: this operator is presented as a phrase adverb modifying the epistemic modality and triggering thus the selection of the mood, indicative or sujbunctive; then its functions within the uterrance are examined. The main aim is, nonetheless, to present the statistics of the use of either indicative or subjunctive mood. The concurrence of both moods has been analyzed on the Spanish synchronic corpus (CREA) and into the analysis all geografical zones included within the corpus have been involved.
\end{abstract}

Keywords (En): modality; Spanish; quizás; indicative; subjunctive; operator; adverb; corpus.

\section{Introducción}

En este artículo ${ }^{1}$ presentamos nuestro análisis del corpus que intenta abarcar el uso del subjuntivo o del indicativo con el operador de modalidad quizá(s). El adverbio quizáa $(s)$ se considera como operador de modalidad epistémica, característica que se refleja en la selección de los modos verbales: el indicativo y el subjuntivo. La opinión más extendida es que el uso del subjuntivo o indicativo deriva de la posición del operador quizás(s), aunque el tema es más complejo, especialmente desde el punto de vista de las intenciones del emisor, dependiendo del grado de la posibilidad o de la hipótesis que el hablante manifieste. ${ }^{2}$ En nuestro análisis, que consideramos como una aproximación introductoria, por basarse en el método cuantitativo, hemos intentado coleccionar datos según los cuales se observaran las preferencias y tendencias en la selección de los modos. Antes de entrar en discusión sobre los resultados del análisis del corpus, consideramos oportuno introducir conceptos claves y el método utilizado en el presente estudio.

\section{Quizá(s), ¿adverbio de frase?}

Tradicionalmente, desde el punto de vista morfológico, quizá(s) se ha clasificado como adverbio de duda. ${ }^{3}$ Sin embargo, dada la evolución de la lingüística en la segunda mitad del siglo XX hacia la pragmática, se le ha etiquetado de operador, denominación que sostenemos aquí por razones expuestas más adelante. Los nuevos enfoques lingüísticos que han dividido el enunciado en la enunciación y en el dictum, y los que han elaborado los conceptos de los actos de habla, han concretizado las funciones de algunos adverbios (y, desde luego, otras expresiones), refiriéndose a ellos como a conectores, operadores, formuladores, atributos oracionales etc. Debido a la disparidad de los

\footnotetext{
${ }^{1}$ Agradezco mucho algunas observaciones que me han sugerido los reseñadores: sus sugerencias he incorporado en el texto.

${ }^{2}$ Desde la perspectiva didáctica, véanse p. ej. las discusiones en los foros en el Centro Virtual Cervantes, accesibles en http://cvc.cervantes.es/foros/leer1.asp?vId=47818.

${ }^{3}$ Quizá(s) se ha merecido la atención en las gramáticas clásicas, como la de GILI GAYA (1961), Alarcos Llorach (1970, 1994), Seco (1982), Gómez Torrego (1999), la R.A.E., Nueva gramática de la lengua española (2010), etc.
} 
enfoques y opiniones, ha surgido una variedad de términos lingüísticos; para dejar clara nuestra posición teórica, nos permitimos una especificación de los conceptos aquí utilizados en relación con la expresión quizá(s).

El adverbio en general, como indica la etimología de la palabra, es un elemento adyacente al verbo (ad-verbum). La función primaria del adverbio (es decir, la función establecida por la tradición como primaria), es la de modificar al verbo (habla descpacio); en segundo grado el adverbio modifica a un adverbio (muy bien) o a un adjetivo (muy bueno). Estas últimas funciones no parecen presentar dificultades de identificación, clasificación y explicación de su comportamiento (semántico, sintáctico o distribucional). Al contrario, es la función primaria del adverbio la que ha despertado el interés lingüístico haciéndonos cuestionar si el adverbio incide sólo en el verbo (núcleo del sintagma verbal) o si envuelve a toda la oración. Ya en las obras hoy clásicas aparece la distinción de las dos funciones: p. ej. para JAKOBSON (1979) los adverbios modifican bien una oración bien un constituyente. Los adverbios que inciden en toda la oración han recibido en las gramáticas tradicionales diferentes nombres: adverbios de frase, atributos oracionales, adverbios oracionales, complementos de frase (en la tradición de la lingüística textual francesa ${ }^{4}$ ). La necesidad de distinguir los adverbios - modificadores del verbo - de los adverbios oracionales se ve justificada por algunas restricciones. Así, como afirma CARBONERO CANO (1980 : 162), algunas de las unidades tradicionalmente consideradas como adverbios modales fallan en las respuestas a preguntas prototípicas: de esta manera es imposible construir la siguiente respuesta:

(1) a. ¿Cómo lo has hecho?

b. *Lo he hecho quizás

La consecuencia de ello es la búsqueda de las posiciones en las que se sitúen estos adverbios oracionales dentro de la estructura oracional, especialmente en el marco de la gramática generativa, por ejemplo, JACKENDOFF (1972), POLLOCK (1989), AlEXIADOU (1997), CinQue (1999), ERNST (1998, 2002), HAIDER (2000), DELFITTO (2005). Se ha observado una diferencia entre los adverbios: los que son capaces de modificar la oración, parecen diferenciarse con una posición interna diferente. Desde el punto distribucional, los adverbios que modifican toda la frase son los que presentan movilidad sin variar el sentido. En este respecto CARBONERO CANO (1980), que habla sobre los formuladores, afirma que mientras que los adverbios modales suelen modificar al núcleo del sintagma, los formuladores envuelven el sintagma más que incidir en un elemento u otro, como en los siguientes ejemplos:

\footnotetext{
${ }^{4}$ Véanse sobre todo Buyssens (1975), Melis (1976), Blanche-Benveniste (1982). Para una revisión profunda de las consideraciones sobre los «adverbios de frase », véase FUENTES RODRIGUEZ (1987).
} 
(2) Formuladores (de duda) - el operador quizá $(s)^{5}$

a. la libertad para Stalin y [ quizás [sN la libertad para los que hoy día...]]

b. creíamos necesaria esta introducción, [ quizás [sA un poco larga,]] ...

c. dicen, [ quizás [sAdv injustamente]], que ...

d. dice, y [ quizás [sp con un punto de razón]], que la moda...

En cuanto a la posición exacta de los adverbios oracionales, lo importante es que se trate de una posición marginal, según un acuerdo general a través de las teorías lingüísticas. La sintaxis textual, teniendo en cuenta el nivel más allá de la oración, propone que el enunciado tenga dos partes: el adverbio y el contenido informado. Así la oración Francamente, no me gusta mucho puede representarse de la siguiente manera:

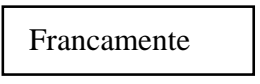

no me gusta mucho

Pero, como afirma FUENTES RodRíGUEZ (1987: 62), « no siempre puede decirse que también sean marginales desde el punto de vista sintáctico y semántico, ya que hay algunos que sí son un argumento del verbo principal ». Sin embargo, los siguientes ejemplos no parecen confirmar que quizá $(s)$ incida sólo en el verbo; al contrario, es difícil, además, adjudicarle una posición dentro de la oración; su posición marginal queda, a nuestro modo de ver, más que clara aun más con la posición del operador parentética:

(4) a. Pero, quizás, estas razones pueden llegar tarde para...6

b. Hermano Mateu admitió que quizás , ,algún desaprensivo“ ha utilizado...

Ahora bien, habida cuenta de la posición marginal del adverbio oracional desde donde influye sobre la oración, se plantea la necesidad de especificar el nivel en el que el operador actúa: en el enunciado o en la enunciación. Dentro de la lingüística se suele discernir, incluyendo al emisor del mensaje, lo enunciado (el dictum) y la enunciación, ${ }^{7}$ por lo tanto los operadores inciden bien en el dictum, bien en la enuncación. ARCE CASTILlo (1998 : 10) lo resume de la siguiente manera:

« Por un lado existen operadores que podríamos incluir dentro de la modalidad de la enunciación, reflejan matices de subjetividad por parte del hablante, son los operadores que denotan deseo, temor o alegría, etc., o que de alguna manera implican al oyente en el acto comunicativo. Y por otro lado pertenecerían a la modalidad del enunciado los operadores que expresan lo verdadero, posible, contingente, dudoso con respecto a lo dicho. »

Mediante los análisis perfomativos, propugnados por Ross (1970), recientemente en BÁEZ SAN JOSÉ (2002), en los que los enunciados en (5) se

\footnotetext{
${ }^{5}$ Todos los ejemplos bajo (3) han sido recogidos en el corpus CREA (www.rae.es), marzo 2012.

${ }^{6}$ Todos los ejemplos bajo (4) han sido recogidos en el corpus CREA (www.rae.es), marzo 2012.

${ }^{7}$ Conf. p. ej. BALLY (1965). Mientras que la mayoría de los autores consultados maneja la dicotomía enunciación :: enunciado (dictum), FUENTES RODRIGUEZ (1991 : 93) propone suponer tres niveles: (i) la enunciación, constitución del acto de decir, (ii) la modalidad, actitud o visión del hablante ante (iii) el dictum, contenido del enunciado.
} 
pueden transformar como está indicado en (6), se puede discernir si el adverbio incide en el dictum o en la enunciación. Así, mientras que el ejemplo (6a) presenta la actitud del hablante ante lo enunciado ( evidentemente »), el adverbio francamente en (6b) indica la actitud del hablante ante la enunciación. Aplicando esta lógica al operador quizá(s), cuyo comportamiento se presenta a continuación en (7), se puede llegar a la conclusión de que este elemento pertenecerá a los operadores del dictum, expresando la actitud del hablante hacia lo dicho:

(5) a. Evidentemente, ya no queda mucho dinero.

b. Francamente, no me gusta nada.

(6) a. [ Digo [que es evidente que ya no queda mucho dinero] ${ }^{\text {DICTUM }}$ ] ENUNCIACIÓN

b. Digo francamente [que no me gusta nada] $\left.{ }^{\text {DICTUM }}\right]^{\text {ENUNCIACIÓN }}$

(7) a. [*digo quizás [ que tienes razón ] $\left.{ }^{\text {DICTUM }}\right]_{\text {ENUNCIACIÓN }}^{\text {ENCTUM }}$

b. [digo [ que quizás tengas razón ] ${ }^{\text {DICTUM }}$ ] $^{\text {ENUNCIACIÓN }}$

c. sucede que yo te digo a ti/Ud. que sucede quizás que tienes razón ${ }^{8}$

\section{Modalidad y significado}

El adverbio quizá(s) como modificador/atributo oracional incide en el sintagma verbal afectando la elección de los modos verbales; ésta es la razón por la cual estos « adverbios » han recibido nombres de operadores de la modalidad, operadores modalizadores, ${ }^{9}$ modalizadores ${ }^{10}$. Aquí no vamos a examinar la diferenciación terminológica de los operadores, marcadores, conectores etc.; ${ }^{11}$ para nuestros fines servirá suficientemente la que sostenemos aquí: quizá(s) es un operador ya que opera sobre el segmento en el sentido de que desencadena reacciones (la selección del modo verbal). ${ }^{12}$

Damos por aclarado que quizá(s) es un operador a nivel del dictum, uno de los operadores de modalidad, de los que ARCE CASTILLO (1998 : 16) afirma que pertenecen a la función representativa, a la modalidad del juicio. Teniendo en cuenta que hay una variedad de opiniones y clasificaciones de la modalidad, podemos atribuirle al elemento quizá $(s)$ la modalidad epistémica (según la división de PERKINS $(1983)^{13}$ y CARRETERo $(1992)^{14}$ ), y/o la extrínseca (según QUIRK et alii (1985), siguiendo a JESPERSEN $(1924,1975))^{15}$. No pretendemos entrar en discusión sobre la modalidad; para los fines de este artículo bastará afirmar que

\footnotetext{
${ }^{8}$ En esta transformación hemos aplicado el test performativo propuesto por BÁEZ SAN JoSÉ (2002 : 78).

${ }^{9}$ Término utilizado en ARCE CASTILlo (1998).

${ }^{10}$ Término utilizado por PORTOLÉs LÁZARO (1993).

${ }^{11}$ Términos que tendían a ser confundidos. Remitimos a revisiones terminológicas en PORTOLES LAZARO (1993), TORDESILLAS (1993), FUENTES RODRIGUEZ (2003).

12 También nos apoyamos en PorTOLEs LAZARO (1993 : 158) para quien el operador proporciona instrucciones que guían las inferencias que se pueden obtener del enunciado en el que aparecen.

${ }^{13}$ Perkins (1983) distingue tres tipos de modalidad según si la posibilidad/necesidad dependan de un juicio (leyes personales), de reglas sociales (leyes sociales) o de las leyes de naturaleza: epistémica, deóntica y dinámica, respectivamente.

${ }^{14}$ CARretero (1992) propone una clasificación de la modalidad incluyendo la noción de la « aceptación».

${ }^{15}$ Estos autores dividen la modalidad en intrínseca y extrínseca según que la posibilidad/necesidad dependan de la voluntad.
} 
mediante la modalidad se expresa la actitud del hablante ante el contenido (ZAVADIL 1980), y como tal es inherente a toda oración, como sostiene por ejemplo ZAVADIL (1968), ${ }^{16}$ y que la modalidad en las lenguas históricas se puede señalar de diferente manera: por el modo verbal, por la entonación, por los recursos lexicales, etc.

En cuanto al « significado » de la modalidad dentro de la cual quizá(s) actúa, situaremos a este operador dentro de la modalidad epistémica en la posición de « lo incierto » (JESPERSEN 1975 : 392, lo positivo, lo incierto, lo negativo). ${ }^{17} \mathrm{Sin}$ embargo, como advierten diferentes autores, el valor de duda puede expresar diferentes matices y existe una gradación de la posibilidad. Afirma CARBONERO CANO (1980 : 173) que « el valor de duda, al encontrarse en un grado cero respecto de la afirmación o la negación, puede quedar matizado hacia una determinada posibilidad de certeza ». Similarmente, CARRETERO (1992 : 52) distingue en el eje de lo epistémico una zona central que equivaldría a la posibilidad, mientras que hacia las zonas del eje más cercanas a los extremos ascendería la probabilidad positiva y negativa. En lo que se refiere a quizá(s) en concreto, CARBONERO CANO (1980 : 173) habla de duda afirmativa y duda negativa; de ahí que clasifique quizás, tal vez y acaso dentro del grupo de los formuladores de duda. En cualquier caso, el significado del elemento en cuestión queda transparente por su etimología: en la pregunta retórica qui sapit ${ }^{18}$ se refleja la duda que, como define MARTín ZORRAQUINO (1999 : 33) « surge en la mente del hablante o que éste adopta cuando la seguridad procede del juicio del autor ». Una definición más detallada se ofrece en VÁZQUEZ PÉREZ (2008), quien incluye a este operador en el grupo de marcadores epistémicos que «indican la decisión que toma el autor del texto de NO comprometerse con la verdad de la proposición expresada ». Para él se trata de un fenómeno de la cortesía negativa, dentro de la cual estos elementos funcionan como minimizadores, que rebajan el compromiso total del autor de la afirmación. Similarmente, ARCE CASTILlO (1998 : 16) define su función como la señal del hablante que pretende suspender la aserción, indicando inseguridad, huir de la responsabilidad, de los juicios emitidos, o mostrarlos como más o menos probables. ${ }^{19}$

\subsection{Funciones de quizás(s) dentro de la oración}

A modo de recapitulación constataremos que consideramos el adverbio quizá(s) como operador, modalizador, que actúa en el nivel del dictum. En el corpus lingüístico español (CREA) hemos detectado, durante nuestro análisis, las siguientes funciones de este elemento, que resumimos a continuación.

\footnotetext{
${ }^{16}$ En concreto, para ZAVADIL « toda oración gramatical indispensablemente tiene que contener la expresión de la modalidad. [...] Esto significa que la modalidad es la categoría fundamental que constituye la predicación » (1968: 63).

17 Para más información sobre las cuestiones de la modalidad, véanse JIMÉNEZ JULIÁ (1989), Fuentes Rodríguez (1991) y CARRETERo (1992), que ofrece una profunda revisión de los conceptos.

${ }^{18}$ Diccionario de la lengua española, vigésima segunda edición, accesible en www.rae.es

${ }^{19}$ Para una discusión sobre valores prototípicos y no prototípicos véase VÁZQUEZ PÉrEZ (2008).
} 


\subsubsection{Modificador del sintagma}

Ya se ha afirmado aquí que una de las funciones de este elemento es la de modificar a un sintagma (SN, SA, SAdv, SP); en estos casos quizá(s) sería un adverbio en el sentido de adjunto $^{20}$ (véanse los ejemplos en (2)). Para identificar qué sintagma es el afectado, CARBONERO CANO (1980 : 164) propone una prueba de la ampliación o transformación teórica de la frase; así quedaría claro el sintagma modificado por quizá(s):

(8) a. Mi hermano quizás trabaje hoy por la noche, o quizás su compañero. ${ }^{21}$

b. Mi hermano quizás trabaje hoy por la noche, o quizás estudie.

c. Mi hermano quizás trabaje hoy por la noche, o quizás mañana por la mañana.

Para los objetivos de nuestro análisis, en los siguientes capítulos nos referiremos a esta función de quizá(s) como al adjunto.

\subsubsection{Operador oracional}

Más interesante para nuestro análisis es el elemento quizá(s) en su función de atributo (adverbio) oracional, o sea del « adverbio de frase $»^{22}$ en el sentido de disjunto. Como tal, quizá(s) actúa como modalizador, es decir operador de modalidad, influyendo directamente la elección del modo subjuntivo o indicativo. Sin embargo, esto no quiere decir que la mera presencia/ausencia afecte la elección del modo verbal; al contrario, es la actitud del hablante la que hace que aparezca un modo u otro. De esta manera la «fuerza » del operador se ve subordinada a las intenciones del emisor: un ejemplo claro es el uso del condicional para expresar la duda (9a) o sugerencia (9b), puesto que en estos contextos se prefiere - según parece - expresar la intención mediante el modo más que mediante un operador que exija el subjuntivo.

(9) a. Antes, quizás faltaría por parte del autor una mejor explicación...$^{23}$

b. Si usted ha marcado una de estas frases, quizás debería comentárselo a su médico.

\subsubsection{Operador oracional único y subordinado}

El factor decisivo para la elección de los modos verbales puede ser o la intención del hablante, cuyo resultado es un enunciado con diferentes grados de duda ante el dictum, o también la presencia de otro operador de rango superior, al que quizá(s) es subordinado. Esto ocurre en oraciones de estructuras canónicas y usos de modos verbales regidos por reglas sintácticas, como en las sustantivas, adjetivas, concesivas, condicionales, etc. Así en (10a) es más que probable que en

\footnotetext{
${ }^{20}$ Según la terminología usada por BeLLERT (1977), los disjuntos indican la actitud del hablante ante lo enunciado, los conjuntos sirven para conectar los enunciados, y los adjuntos modifican un elemento en concreto.

${ }^{21}$ Los ejemplos están tomados de CARBonero CANO (1980 : 164).

${ }^{22}$ A modo de resumen citaremos la definición de FuenTES RodRíGuez (1987 : 67): « Se le llama 'adverbio de frase' a todo aquello (unidad o locución) que no tiene una función modificadora de un elemento concreto, sea adjetivo, verbo o adverbio, los tres tipos de incidencia reconocidos por la tradición, ni a un sintagma nominal. Por el contrario, se refiere a toda la oración. Tiene movilidad generalmente y suele ir entre pausas ».

${ }^{23}$ Todos los ejemplos bajo (9) han sido recogidos en el corpus CREA (www.rae.es).
} 
el subjuntivo participe el significado de la conjunción aunque; el condicional en (10b) parece regido por la regla de las condicionales hipotéticas, mientras que el subjuntivo en (10c) parece justificado por la lectura especificativa e hipotética de la adjetiva/relativa:

(10) a. La pregunta es apasionante aunque quizás esto de empezar y acabar no fuesen más que... ${ }^{24}$

b. Si estos otros agentes no estuvieran en ARCO quizás el acontecimiento ferial se restringiría sólo a su vertiente económica, ...

c. Los resultados de la estimulación eléctrica sobre la enfermedad vascular periférica deben ser objeto de una valoración específica atendiendo a la evolución de la lesin isquémica, sobre la que quizás otros estudios abran expectivas más alentadores.

Para los objetivos de nuestro análisis, en los siguientes capítulos nos referiremos a esta función de quizá(s) como al operador subordinado mientras que en los casos donde un operador superior es ausente, como al operador único.

\section{Análisis del corpus}

En esta parte vamos a presentar los resultados obtenidos en el corpus mediante un análisis realizado entre diciembre de 2010 y julio de 2012, cuyo objetivo ha sido cuantificar los diferentes modos verbales en enunciados que contenían el operador quizá(s). ${ }^{25}$ Se ha trabajado con el corpus CREA, dividiendo la muestra en zonas geográficas: España, América Latina - zonas mejicana, central, caribe, andina, chilena, y rioplatense.

Para la división en las zonas hemos seguido la propuesta del corpus CREA según la tabla en (11), partiendo de la hipótesis de que tal división permitirá captar agentes diatópicos. Dentro de cada zona se ha trabajado con los países correspondientes, es decir, la consulta se ha realizado país por país (lo que permite el sistema de búsqueda). ${ }^{26}$

(11) Distribución de textos en el corpus CREA

\begin{tabular}{|l|l|r|l|}
\hline España & América & \multicolumn{2}{|c|}{$50 \%$} \\
\hline \multirow{5}{*}{$50 \%$} & $\begin{array}{l}\text { zona } \\
\text { mexicana }\end{array}$ & $40 \%$ & $\begin{array}{l}\text { México, Sudoeste de Estados Unidos, Guatemala, Honduras, El } \\
\text { Salvador }\end{array}$ \\
\cline { 2 - 4 } & zona central & $3 \%$ & Nicaragua y Costa Rica \\
\cline { 2 - 4 } & zona caribeña & $17 \%$ & $\begin{array}{l}\text { Cuba, Puerto Rico, Panamá, Rep. Dominicana, costas de } \\
\text { Venezuela y Colombia y nordeste de Estados Unidos }\end{array}$ \\
\cline { 2 - 4 } & zona andina & $20 \%$ & resto de Venezuela y Colombia, ${ }^{27}$ Perú, Bolivia y Ecuador \\
\cline { 2 - 4 } & zona chilena & $6 \%$ & Chile \\
\cline { 2 - 4 } & $\begin{array}{l}\text { zona } \\
\text { rioplatense }\end{array}$ & $14 \%$ & Argentina, Paraguay y Uruguay \\
\hline
\end{tabular}

\footnotetext{
${ }^{24}$ Todos los ejemplos bajo (10) han sido recogidos en el corpus CREA (www.rae.es).

${ }^{25}$ Trabajos de investigación presentados como tesis de fin de carrera: DrAŠAROVÁ (2011), HAVlíkOVÁ (2012), KunZ (2012), PAPEŽovÁ (2012), completados por un análisis adicional que abarcó las zonas no observadas en dichas tesis, realizado por la autora del presente artículo.

${ }^{26}$ Aunque la consulta por PAÍS en realidad no siempre equivale al origen del autor, sino señala solamente el lugar de la publicación del texto.

${ }^{27}$ Dado que el sistema de consulta en el corpus permite seleccionar solo países enteros, por lo cual no es posible separar zonas de los países, hemos incluido todas las muestras obtenidas de Venezuela y Colombia en la zona andina.
} 
Los análisis parciales han seguido pasos unificados que explicamos a continuación. Para cada zona geográfica (i.e. para cada país de la zona) se han buscado formas quizás y quizá para obtener enunciados con el operador en posición no inicial, y Quizás y Quizá, para posiciones iniciales, es decir, al principo del enunciado. Las apariciones obtenidas se han reducido a números cómodos para analizar, alrededor de unas 70 o 80 muestras como mínimo (aunque en el caso de algunos países/zonas las apariciones eran muy bajas; en estos casos no se ha utilizado, lógicamente, ningún filtro). Dado que para cada zona (país) se han obtenido resultados en cantidades diferentes en cuanto al número total, habiendo apariciones muy variadas para cada país, éstas se han contado en valores relativos, en porcentajes, para posibilitar las subsecuentes comparaciones

A continuación, se han descartado casos en los cuales quizá(s) cumple la función de adjunto (12a) para obtener enunciados con operador oracional (los enunciados con adjuntos forman un 51\% de los casos obtenidos). También se han dejado de lado los casos del llamado operador subordinado, que formaron un $9 \%$ de la muestra total (12b).

(12) a. será probablemente reinhumado en Espña, quizás temporalmente, pues Daniel Paladino, líder...

b. aunque temen que quizás sea tarde...

En la muestra de los operadores oracionales se han observado los modos en relación con la posición del operador en la oración y la posición mutua del operador y verbo: (a) posición inicial del operador, (b) posición media del operador seguido del verbo, y (c) posición media del operador precedido por el verbo. En cuanto a la distancia entre el operador y el verbo, no se ha identificado una tendencia marcada en la preferencia del modo; el subjuntivo y el indicativo aparecen tanto en posiciones inmediatas (13) como en las discontinuas (14). Sin embargo, en nuestra opinión, este aspecto se merecería un análisis más detallado.

(13) a. ...y por eso quizá resulte sensato...

b. Esto quizás es uno de los factores...

(14) a. Quizás [ lo más importante [ del documento]] descanse en el...

b. y quizás [ los antiguos médicos europeos ] [ también ] estaban...

c. Quizás [ para el año [que viene]] estemos hablando

d. Quizás [ en otro momento [cuando ambos se calmen ]] pueda ...

\section{Estadísticas}

\subsection{España}

Como podemos observar en el gráfico 1, las tendencias en el uso de los modos son más o menos equilibradas. La única discrepancia ha surgido en relación con la posición del operador pospuesto (minoritaria por lo general), para la cual no se han identificado casos con subjuntivo. En realidad, entre las muestras ha aparecido la secuencia <subjuntivo; quizás>, sin embargo la presencia del modo subjuntivo la hemos adjudicado a un elemento subordinante; de ahí que quizás sea un operador subordinado y que el subjuntivo esté regido por reglas sintácticas: 
(15) a. Por otra parte, no es un texto antiguo, es actual. Y es que según avanza esta sociedad consumista en la que vive buena parte del mundo, los sentimientos hacia nuestro entorno se vuelven cada vez más despreocupados. Por ello es que un texto como éste no hubiese sido necesario quizás en épocas más antiguas, sino actuales,...

b. En primavera, el comportamiento de las aves hace que la observación del paso sea quizás más complicada que en otoño,...

Si sumáramos la mera ocurrencia de las formas del subjuntivo y el indicativo, llegaríamos a la prevalencia del indicativo $(59,5 \%)$ sobre el subjuntivo $(40,5 \%)$. Sin embargo, si tomamos en cuenta las posiciones del operador y el verbo, llegamos a cantidades algo diferentes. De los resultados se deduce, pues, una preferencia por el indicativo en las secuencias <quizá(s) inicial; verbo> y <verbo; quizá $(s)>$. Por su parte, el subjuntivo tiende a aparecer si el operador precede al verbo en la posición media, lo cual podríamos formular como una tendencia relacionada con la posición mutua del verbo y el operador: el operador antepuesto contribuye a la aparición del subjuntivo. Sin embargo, los resultados parciales del corpus de España no parecen confirmar tal conclusión, ya que se ha identificado una preferencia, aunque ligera, por el indicativo en la secuencia <operador; verbo> en posiciones no iniciales de la oración.

\section{Gráfico 1: España}

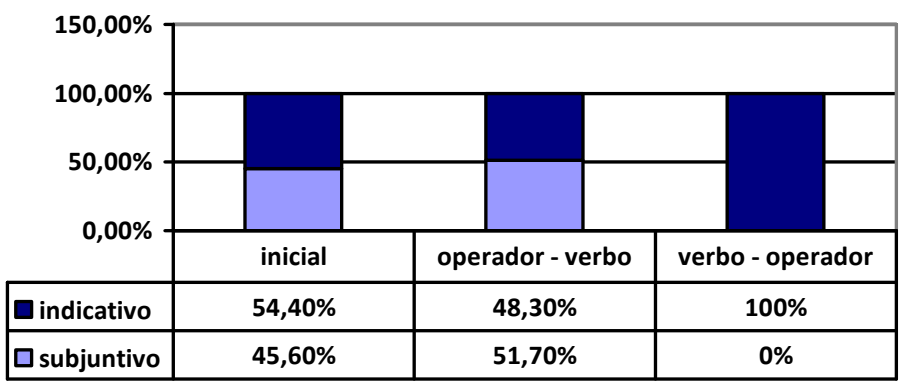

(16) Ejemplos de las ocurrencias en las posiciones observadas:

a. Quizás sea una forma de enuciar el principio de Hamilton

b. Quizás ha faltado más tiempo para los debates.

c. En consecuencia, quizá haya llegado el momento de replantearse...

d. ... cómo aquellos pobres actores, que quizás habían sufrido mucho para llegar...

e. El mildiu (Plasmophora viticola) es quizás el hongo que más problemas causa...

El porcentaje subido en el indicativo después del operador en la posición inicial nos ha parecido interesante, ya que para esta posición esperábamos un número mayor del subjuntivo; por lo cual hemos realizado un análisis parcial para ver la distribución indicativo-subjuntivo en los diferentes medios y para ver si la distribución está - y hasta qué punto - relacionada con el tipo de textos. De hecho, mientras que en revistas, libros y periódicos los modos están relativamente equilibrados en cuanto a su distribución numérica, con un 51,25\%, 52,4\% y 53,5\% para el indicativo respectivamente, en miscelánea y oral aumentan las 
preferencias: $54,25 \%$ para el subjuntivo en miscelánea y $63,5 \%$ para el indicativo en el corpus oral (véase gráfico 2).

\section{Gráfico 2: España, Quizá(s) inicial, medios}

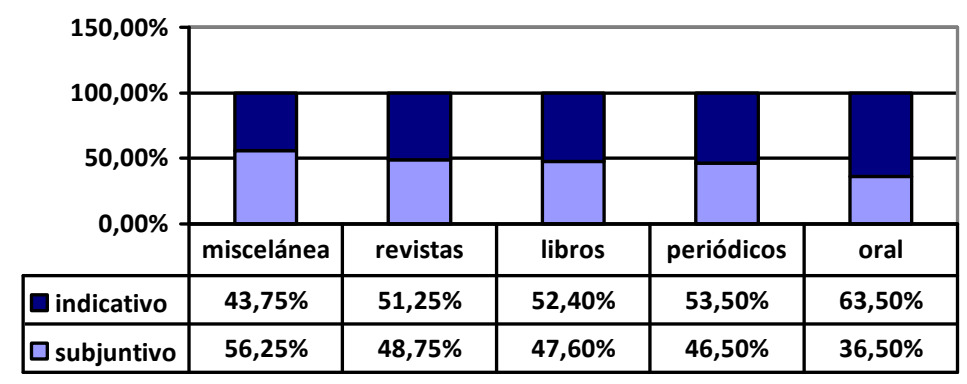

\subsection{América Latina}

El primer análisis de los datos de España nos ha llevado a las siguientes conclusiones: verbo>;

(i) preferencia por el indicativo en las secuencias <quizá(s) inicial,

(ii) preferencia por el indicativo en las secuencias 〈verbo, quizá(s)>;

(iii)preferencia por el subjuntivo en las secuencias <quizá(s) medio, verbo>.

En lo que sigue queremos comparar estos resultados con los de las zonas de América Latina. En cuanto a la posición inicial del operador, todas las zonas confirman la conclusión (i), con un número de ocurrencias relativo aun más elevado; sobre todo en las zonas chilena, central y caribe, con un 67,6\%, 65,2\% y $65,2 \%$ respectivamente. En este caso, pues, se muestra una tendencia general de la preferencia por el indicativo más marcada que en España (gráfico 3).

Por el contrario, la conclusión (iii) sobre la preferencia por el subjuntivo en la secuencia <quizá(s), verbo〉, ocupando el operador una posición no inicial del enunciado, se ha negado completamente: en el corpus de América Latina se resaltan tendencias contrarias, es decir, prevalece el uso de indicativo en todas las zonas observadas, destacando con números mayores las zonas chilena y central, con un $68,75 \%$ y $66,5 \%$ respectivamente. Como en el caso anterior, también en este punto se puede observar una tendencia hacia el uso del indicativo en América Latina (gráfico 4).

En lo que se refiere a la conclusión (ii), que formula el uso (casi) absoluto del indicativo si el verbo precede al operador en posición media, las ocurrencias en todas las zonas de América Latina comprueban tal tendencia. Sin embargo, los resultados, que en este caso han alcanzado hasta el 100\% de ocurrencias, se deben a apariciones extremamente escasas en algunos países observados, que no superaban uno o dos casos obtenidos en el corpus (gráfico 5). 
Dejando aparte esta tercera posición observada, podemos concluir que hay una clara preferencia por el indicativo en las zonas latinoamericanas frente al español europeo. Además, esta tendencia se manifiesta con diferente fuerza en las zonas: así, mientras que en los resultados obtenidos para España las diferencias en el eje indicativo-subjuntivo son casi insignificantes, en América Latina, por lo general, las tendencias hacia el uso del indicativo son más marcadas, aunque con diferente fuerza en diferentes zonas. Igual que en el español peninsular con usos relativamente equilibrados (conf. $54,4 \%$ vs. 45,6\% para la posición inicial del operador, y $48,3 \%$ vs. $51,7 \%$ para las posiciones medias), los resultados con pocas diferencias se muestran en la zona rioplatense (conf. 54,1\% vs. 54,9\%, y 51,3\% vs. $48,7 \%$, respectivamente). Por el contrario, las zonas con diferencias más marcadas son la mexicana (conf. $68,9 \%$ vs. $31,1 \%$ ), la chilena (conf. $68,75 \%$ vs. $31,25 \%$ ) y la central (conf. $66,5 \%$ vs. $34,8 \%$ ), seguidas por la zona caribe. En una escala se situarían de la siguiente manera: zona mexicana $>$ chilena $>$ central $>$ caribe $>$ andina > rioplatense/España.

También es interesante observar los usos de los tiempos, tanto en subjuntivo como en indicativo. De los resultados, recogidos en el gráfico 6, se deduce que la categoría modotemporal más productiva es el presente de subjuntivo con un $36,20 \%$ de ocurrencias, seguido por el presente de indicativo con un $23,96 \%$; en el segundo extremo del eje se ha situado el futuro con un $1,30 \%$ y el pluscuamperfecto de indicativo con un $0,52 \%$.

\section{Gráfico 3: Quizá(s) en posición inicial}

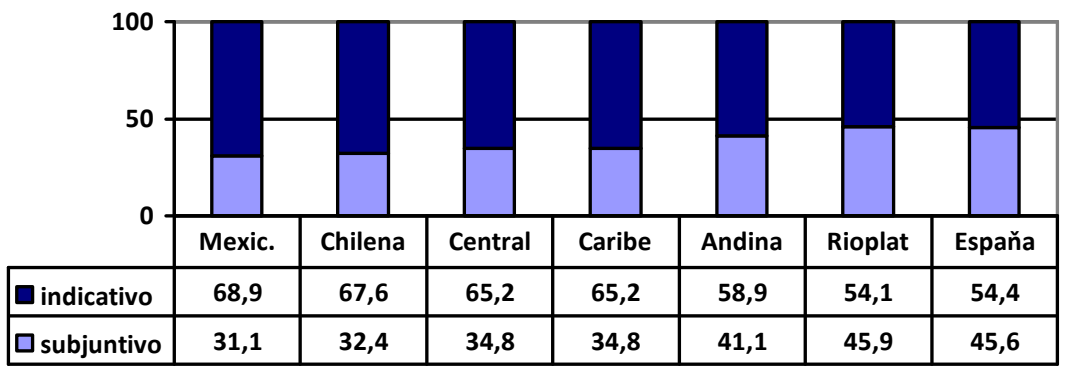

\section{Gráfico 4: Operador quizá(s) - verbo}

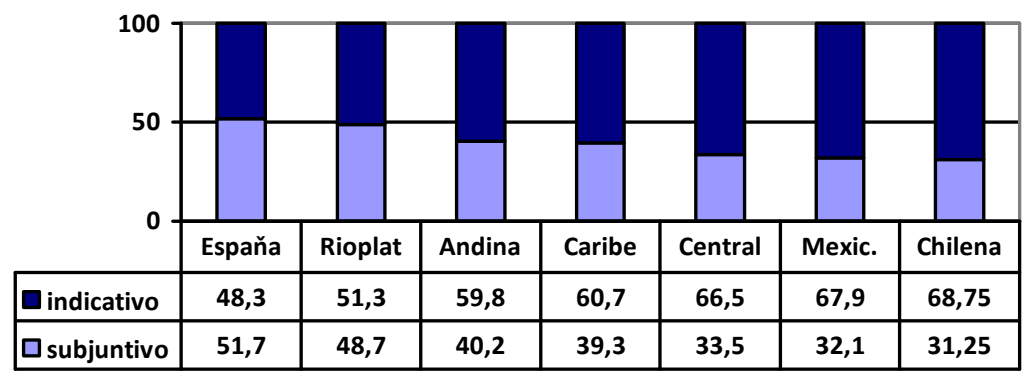


Gráfico 5: verbo - operador quizá(s)

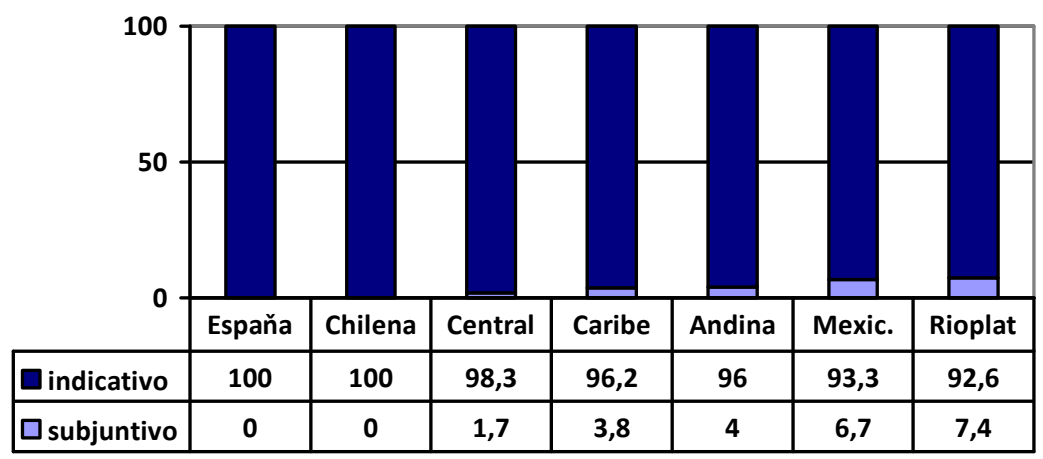

\section{Gráfico 6: Distrubución de las categorías modotemporales}

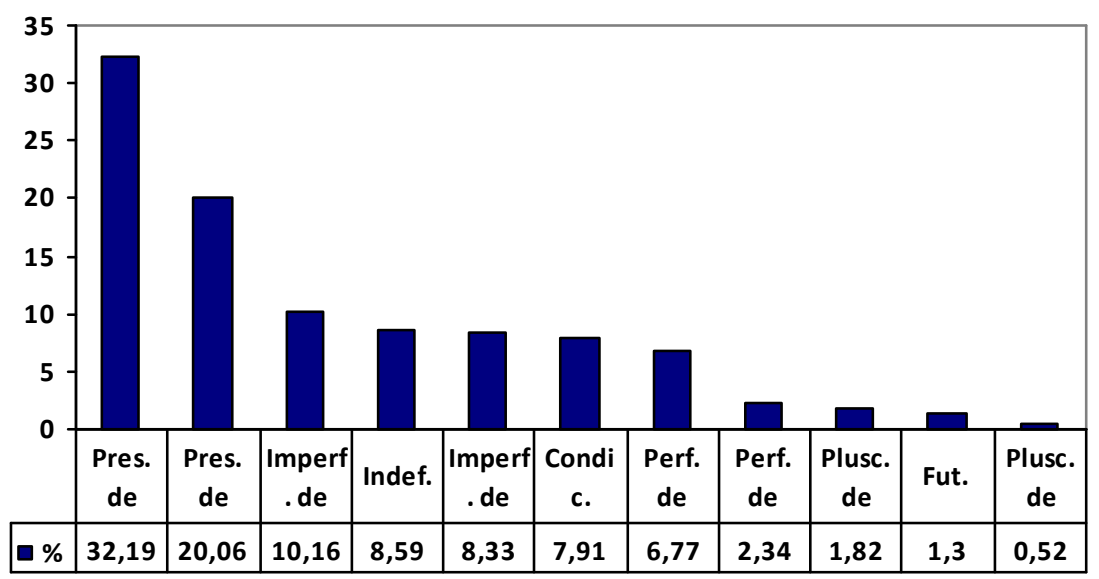

\section{A modo de conclusión}

En el presente artículo hemos intentado ofrecer, después de una parte introductoria sobre el estatus del operador oracional quizá(s), un análisis cuantitativo que recoge el uso del subjuntivo/indicativo con dicho operador. Con el análisis, que abarca las ocurrencias en el corpus CREA, tanto para el español europeo como para el(los) latinoamericano(s), hemos querido cuantificar los diferentes usos recogidos en el corpus y formular tendencias, por lo menos numéricas, divididas (a) por zonas y (b) por posiciones mutuas del operador y el verbo. De esta manera hemos llegado a las siguientes conclusiones:

1. Los usos del subjuntivo/indicativo no muestran considerables diferencias.

2. Si hay diferencias en dicho uso, las más marcadas aparecen en América Latina (especialmente en las zonas chilena, central y caribe). 
3. La posición mutua del verbo y del operador influye en el uso del subjuntivo o indicativo.

4. Si el operador precede al verbo, la tendencia es usar el indicativo.

5. Si el operador está en posición inicial, la tendencia es usar el indicativo.

6. Si el operador está en posición media, la tendencia es usar el indicativo, con exepción del español peninsular.

7. Si el verbo precede al operdor, la tendencia hacia usar el indicativo es muy marcada.

Sin embargo, somos conscientes de que no se trata sino de una aproximación cuantitativa, que debería ser seguida con un análisis cualitativo y profundo. Así, hemos intentado esbozar una línea posible, aunque dentro del marco del método cuantitativo, enfocada hacia los medios (cap. 5.1.), pero se ofrecen otros aspectos a estudiar, como autores, temas, etc., en fin, lo que permite el trabajo con el corpus CREA. Y, sobre todo y apesar de todo esto, somos conscientes de que la decisión del emisor por usar un modo u otro está más allá de cualquier método cuantitativo. Por otro lado, creemos que con este análisis hemos contribuido con un primer paso, a partir del cual se pueden realizar otros estudios concernientes al tema.

\section{BIBLIOGRAFÍA}

AlARCOS LlORACH Emilio (1970), Estudios de gramática funcional del español, Madrid, Gredos.

Alarcos LloRACH Emilio (1994), Gramática de la lengua española, RAE, Colección Nebrija y Bello, Madrid, Espasa.

AleXIAdou Artemis (1997), Adverb Placement: A Case Study in Antisymmetric Syntax, Amsterdam, Benjamins.

ARCE CASTILlo Ángela (1998), Los conectores pragmáticos como índices de modalidad en español actual, E.L.U.A., 12, p. 9-23.

BALly Charles (1965), Linguistique générale et linguistique français, Berne, Francke.

BELLERT Irena (1977), On semantic and distribucional properties of sentential adverbs, Linguistic Inquiry, 8, 2, p. 337-351.

BLANCHE-BENVENISTE Claire (1982), Verb complements and sentence complements: two different types of relation, Communication and cognition, 15, 3/4, p. 333-361.

BUYSSENS Eric (1975), La classification des adverbes, Revue Roumaine de Linguistique, 20, p. 461-463.

CARBOnero CANO Pedro (1980), Afirmación, negación, duda, Revista española de lingüística, 10, 1, p. 161-176.

CARretero Marta (1992), Una propuesta de tipología de la modalidad: la aceptación como categoría modal, DICENDA. Cuadernos de Filología Hispánica, 10, p. 41-61.

CInQue Guglielmo (1999), Adverbs and Functional Heads. A Cross-linguistic Perspective, New York, Oxford University Press.

DELFITTO Denis (2005), Adverb classes and adverb placement, in : M. EvERAERT, H. van RIEMSDIJK (eds.), Blackwell Companion to Syntax, Oxford, OUP, p. 83-120. 
DRAŠAROVÁ Lenka (2011), Používání subjuntivu/indikativu po operátorech pravěpodobnostní modality v jihoamerické španělštině - oblast centrální, mexická a karibská (El uso del subjuntivo/indicativo con operadores de modalidad de probabilidad en el español latinoamericano - zona central, mexicana y caribeña). Thesis, Jihočeská Univerzita, České Budějovice.

ERnst Thomas (1998), Scope based adjunct licensing, in : P. TAMANJI, K. Kusumoto (eds.), Proceedings of the North East Linguistics Society (NELS 28), Amherst, GLSA, p. 127-142.

ERnST Thomas (2002), The Syntax of Adjuncts. Cambridge, CUP.

FUENTES RODRÍGUEZ Catalina (1987), El adverbio de la frase, RESLA, 3, p.55-74.

FUENTES RODRÍGUEZ Catalina (1991), Algunas reflexiones sobre el concepto de modalidad, RESLA, 7, p .93-108.

FUENTES RODRÍGUEZ Catalina (2003), Operador/conector, un criterio para la sintaxis discursiva, RILCE, 19, 1, p. 61-85.

GILI GAYA Samuel $\left(1961^{8}\right)$, Curso superior de sintaxis española, Barcelona, Spes.

GóMEZ TORREGo Leonardo (1999), Gramática didáctica del español, Madrid, SM.

HAIDER Hubert (2000), Adverb placement: Convergence of structure and licensing, Theoretical Linguistics 26, 1-2, p. 95-134.

HAVlíKová Martina (2012), Použivání subjuntivu/indikativu po operátorech pravěpodobnostní modality (quizás v neiniciální pozici) v evropské španélštině - (El uso del subjuntivo/indicativo con operadores de modalidad de probabilidad (quizás en posiciones no incicales) en el español europeo), Thesis, Jihočeská Univerzita, České Budějovice.

JACKENDOFF Ray (1972), Semantic interpretation in generative grammar, Cambridge, MA, MIT Press.

JESPERSEN Otto (1975), La filosofía de la gramática, (edición española, orig. 1924 The Philosophy of Grammar), Barcelona, Anagrama.

JiMÉNEZ JULIÁ Tomás (1989), Modalidad, modo verbal y modus clausal en español, Verba, 16, p. 175-214.

KunZ David (2012), Použivání subjuntivu/indikativu po operátorech pravěpodobnostní modality (quizás) v jihoamerické španělštině: oblast andská, chilská a Río de la Plata - (El uso del subjuntivo/indicativo con operadores de modalidad de probabilidad (quizás) en el español latinoamericano: zonas andina, chilena y rioplatense), Thesis, Jihočeská Univerzita, České Budějovice.

MELIS Ludo (1979), Compléments de phrase et compléments transpropositionnels, Travaux de Linguistique, 6, p. 13-31.

PAPEŽOVÁ Michaela (2012), Používání subjuntivu/indikativu po operátorech pravěpodobnostní modality (quizás v iniciální pozici) v evropské španělštině (El uso del subjuntivo/indicativo con operadores de modalidad de probabilidad (quizás en posiciones incicales) en el español europeo), Thesis, Jihočeská Univerzita, České Budějovice.

PERKINS Michael R. (1983), Modal Expressions in English, London, Frances Pinter.

Pollock Jean-Yves (1989), Verb Movement, UG, and the Structure of IP, Linguistic Inquiry 20, p. 365-424. 
PORTOLÉs LÁZARO José (1993), La distinción entre los conectores y otros marcadores del discurso en español, Verba, 20, p. 141-170.

SECO Rafael (1982), Manual de gramática española, Madrid, Aguilar.

R.A.E. (2010), Nueva gramática de la lengua española, Madrid, Espasa.

Ross John R. (1970), On declarative sentences, in : R.A. JACOBS, P.S. ROSENBAUM (eds.), Readings in English Transformational Grammar, Walthan, Mass., Ginn and company, p. 222-272.

TORDESILlas Marta Inés (1993), Conectores y operadores: una diferencia de dinámica argumentativa, Revista de Filología Francesa, 3, p. 233-244.

VÁZQUEZ PÉREZ José A. (2008), Mecanismos de atenuación en español e italiano: quizá y forse, redELE, Revista electrónica de Didáctica ELE, 12. Accesible en: <http://www.redined.mec.es/opac/mostrar.php?registro=52970>

ZAVADIL Bohumil (1968), Medios expresivos de la categoría de modalidad en español, Ibero-americana Pragensia, 2, p. 57-86.

ZAVADIL Bohumil (1980), Kategorie modality ve španělštině, Praha, Univerzita Karlova. 